\title{
The Effect of the Operator Learning Curve on Diagnostic Accuracy for Lung Cancer with Endobronchial Ultrasound Guided Transbronchial Needle Aspiration (EBUS-TBNA): A Single-Center Observational Study
}

\author{
Rusen UZUN ${ }^{1}$, Fatih UZER ${ }^{2}$ \\ ${ }^{1}$ Antalya Training and Research Hospital, Department of Respiratory Diseases, Antalya \\ ${ }^{2}$ Kastamonu State Hospital, Department of Respiratory Diseases, Kastamonu, TURKEY
}

\begin{abstract}
We aimed to investigated the relationship between diagnostic accuracy of ultrasound-guided trans-bronchial needle aspiration (EBUSTBNA) and the number of procedure performed by operator. We included all consecutively attended patients undergoing EBUS-TBNA for diagnosis of lung cancer. The pathology prearations were evaluated by the same single pathologist. Rapid on-site examination (ROSE) was performed for all patients. To determine the impact of operator experience on the accuracy of EBUS-TBNA-based diagnosis, patients were divided into three equally sized groups based on the chronological order of their EBUS-TBNA assessment. A total of 270 patients were included in the study. The most common indication for undergoing the EBUS-TBNA procedure was tumor staging (44.1\%). ROC analysis revealed that the study bronchoscopist's diagnosis of malignancy based on EBUS-TBNA images were consistent with the final pathologist's diagnosis in $73 \%$ of the first 90 cases $(p=0.02), 90 \%$ of the second 90 cases $(p=0.01)$, and $98 \%$ of the third 90 cases $(p=0.01)$. In the first 90 cases, the sensitivity of the bronchoscopist's diagnosis was $74 \%$, the specificity was $78 \%$, the positive predictive value was $76 \%$, and the negative predictive value was $85 \%$. In the second 90 cases, the sensitivity was $94 \%$, the specificity was $92 \%$, the positive predictive value was $95 \%$, and the negative predictive value was $95 \%$. In the third 90 cases, the sensitivity was $98 \%$, the specificity was $98 \%$, the positive predictive value was $98 \%$, and the negative predictive value was $100 \%$. We observed that as the number of EBUS-TBNA procedures that the operator performed increased, his diagnostic accuracy approached perfection.
\end{abstract}

Keywords: EBUS, Learning curve, Lung cancer, Diagnostic accuracy

\section{INTRODUCTION}

Lung cancer is one of the most common cancers, with an estimated 2.1 million new cases worldwide in 2018. ${ }^{1}$ In recent years, endobronchial ultrasound-guided transbronchial needle aspiration (EBUS-TBNA) has become the most commonly used method for diagnosis and staging of lung cancer. ${ }^{2}$ EBUS-TBNA is less invasive and has lower morbidity and mortality rates, compared to mediastinoscopy. ${ }^{3}$ Compared to another diagnostic method, has conventional TBNA, it has higher diagnostis and accuracy rates, since EBUS-TBNA provides the chance of simultaneous imaging. ${ }^{4}$ EBUS-TBNA is an operator-dependent procedure for which diagnostic accuracy may increase as the knowledge and experience of the operator increases. ${ }^{2,5}$

Few studies report the association between the knowledge and experience of the operator and diagnostic accuracy of EBUS-TBNA. Further, evidence varies regarding the number of procedures needed for an operator to gain mastery of diagnostic methods similar to EBUS-TBNA. 
Before the widespread use of convex probe endobronchial ultrasound (CP-EBUS), which enables real-time imaging guidance in obtaining samples, the American College of Chest Physician (ACCP) recommended that operators perform at least 50 radial probe endobronchial ultrasound (radialEBUS) procedures before undertaking lung cancer diagnosis in patients. ${ }^{6}$ Another study showed that operators cannot make diagnoses before performing at least 20 EBUS procedures, also the rate of accurate diagnosis does not increase until operators perform at least 50 procedures. ${ }^{7}$ In a study by Kemp et al. which assessed five EBUS operators, diagnostic accuracy increased only after they had performed at least 100 procedures. $^{3}$ Thus, it is unclear how manyprocedures operators need to perform before they can reliably and accurately diagnose lung cancer with EBUS-TBNA. Using data from a training and research hospital,we investigated the relationship between diagnostic accuracy of EBUS-TBNA and the number of procedure performed by an operator.

\section{PATIENTS AND METHODS}

\section{Patients}

In this observational study, we included all consecutively attended patients undergoing EBUS-TBNA for diagnosis of lung cancer at the Antalya Training and Research Hospital between 1 March 2018, and 31 December 2019. Information on patient sociodemographic factors and EBUS-TBNA findings, including mediastinal lymph node distribution and final pathological diagnoses, were recorded in a standardized paper-based data form. During the EBUS-TBNA procedure, both computed tomography and ultrasonography were used to record the short diameters of the lymph nodes. EBUSTBNA of all patients was performed by the same operator, a certified bronchoscopist, and all cytology preparations were examined by a single cytopathologist. Rapid on-site examination (ROSE) was done for all patients. The accuracy of EBUSTBNA diagnosis was determined by comparing the bronchoscopist's diagnosis to the final pathological diagnosis. To determine the impact of operator experience on the accuracy of EBUS-TBNA-based diagnosis, patients were divided into three equal- ly sized groups based on the chronological order of their EBUS-TBNA assessment. This chronological order correlated with the number of prior EBUS-TBNA procedures that the bronchoscopist had performed, and thus the later patient groups represent diagnoses made by a more experienced operator. Using the pathologist's diagnosis as the gold standard, we calculated the accuracy rate, sensitivity and specificity ratio, positive predictive value, and negative predictive value and compared them across the three patient groups. Informed consent was obtained from all patients before any study procedures were performed. Approval for the study protocol was obtained from the Clinical Studies Ethical Committee of Antalya Training and Research Hospital.

\section{Radiological Evaluation}

Contrast thorax tomography (CT) and/or Positron Emission Tomography (PET-CT) were conducted in all patients. The EBUS-TBNA procedure was conducted in patients with mediastinal hilar lymph nodes with a short axis of $>10 \mathrm{~mm}$ and/or a maximum standardized uptake (SUV $\max$ ) value of $>2.5$ based on CT and/or PET-CT.

\section{EBUS-TBNA Procedure}

All EBUS-TBNA procedures were conducted in a dedicated procedure room using a Fujinon EBUS device (7.5 MHz EB-530US/Sonart SU-1, Tokyo, Japan) on patients under conscious sedation with midazolam plus propofol. Lymph node sampling was conducted using a 21-gauge (NA-201SX-4021; Olympus) or 22-gauge (NA-201SX-4022; Olympus) needle. Mediastinal lymph nodes were defined according to the Association for the Study of Lung Cancer (IASLC) criteria. ${ }^{8}$ In patients with suspected benign disease, at least three samples were taken from at least two lymph node stations. For tumor staging in patients with suspected malignant disease, all the stations were scanned starting from the N3 lymph node, and at least three samples were taken from each lymph node station. In patients with a diagnosis of lung cancer, at least four samples were taken from each station for further molecular tests. 


\begin{tabular}{|ll|}
\hline Table 1. Patient characteristics & \\
\hline Age (years \pm SD) & $62.0 \pm 10.3$ \\
Female gender, $\mathrm{n}(\%)$ & $73(27 \%)$ \\
Short axis diameter on EBUS, $\mathrm{mm} \pm \mathrm{SD}$ & $21 \pm 8.8$ \\
Short axis diameter on CT, mm \pm SD & $20.2 \pm 8.5$ \\
Presence of endobronchial lesion, $\mathrm{n}(\%)$ & $40(14.8 \%)$ \\
Presence of a mass in the lungs on CT, $\mathrm{n}(\%)$ & $158(58.5 \%)$ \\
Number of sampling & $3.6 \pm 1.0$ \\
Lymph node Suv max & $8.8 \pm 5.2$ \\
Indication for EBUS-TBNA & \\
Diagnosis, $\mathrm{n}$ (\%) & $80(29,6)$ \\
Staging, $\mathrm{n}$ (\%) & $119(44,1)$ \\
Diagnosis and Staging, $\mathrm{n}(\%)$ & $8(3)$ \\
Studying Molecular Test, $\mathrm{n}(\%)$ & $11(4.1)$ \\
Extra-thoracic Metastasis of malignancy, $\mathrm{n}(\%)$ & $46(17)$ \\
Recurrence / Re-Staging, $\mathrm{n}(\%)$ & $6(2.2)$ \\
\hline
\end{tabular}

\section{Pathological Evaluation}

A section of the cytological material obtained by fine need aspirate was prepared as a cytopathological smear and stained with Diff-Quik for rapid evaluation of the patient. The remaining material was prepared as a cell block using a cell protective solution ( $10 \%$ formaldehyde). Cytopathological evaluation of cell blocks was performed for patient diagnosis using the following categories: (1) non-diagnostic cytology; (2) benign cytology; and (3) malignant cytology, sub-categorized as (a) adenocarcinoma, (b) squamous cell carcinoma, (c) non-small-cell carcinoma, unclassifiable, and (d) small-cell carcinoma.

\section{Patient Follow-Up}

Lymph nodes were accepted as benign if no tumoral cells were observed on EBUS-TBNA confirmed histopathologically with lymph node dissection or in cases where no disease progression was observed clinically and radiologically in at least 6 months of follow up. Malignant lymph node diagnosis was based on the visualisation of tumoral cells on EBUS-TBNA or the histological results of surgical excision.

\section{Statistical Analysis}

Descriptive statistics, including frequencies, means, and percentages were used to evaluate pa- tient demographics, diagnoses, and tumor staging. Differences in characteristics between patients groups were evaluated using chi-square tests for categorical variables and independent Student's ttests for continuous variables. A receiver-operating characteristic (ROC) analysis was performed to examine the consistency of the diagnostic efficacy evaluations of the study groups. AUROC (Area Under the Receiver Operating Characteristic) values were used to compare the ROC curves. Power analysis was done for determining sample size of groups. In all statistical analyses, we used SPSS Statistics for Windows, Version 19.0 (IBM Corp. Armonk, NY, USA), and performed statistical tests using $\alpha=0.05$ as the critical value.

\section{RESULTS}

\section{Patient Characteristics}

From 1 March 2018 to 31 December 2019, 276 patients underwent EBUS-TBNA for lung cancer assessment at the Antalya Training and Research Hospital. Six patients with missing data in their clinical files were excluded from the study. A total of 270 patients were included in the study, among whom 73 (27\%) were female and $197(73 \%)$ were male; the mean patient age was 62.0 years \pm 10.3 (Table 1). During CT evaluation, a mass lesion was detected in the lung in 158 (58.5\%) patients, and an 
International Journal of Hematology and Oncology

Table 2. Evaluation of measurements according to case groups

Measurement

Case Groups

\begin{tabular}{|c|c|c|c|c|}
\hline & $\begin{array}{l}\text { Cases } 1-90 \\
(n=90) X \pm S D\end{array}$ & $\begin{array}{l}\text { Cases } 91-180 \\
(n=90) X \pm S D\end{array}$ & $\begin{array}{l}\text { Cases 181-270 } \\
(n=90) X \pm S D\end{array}$ & $\mathbf{p}$ \\
\hline Age $61.5 \pm 10.7$ & $62.9 \pm 10.4$ & $61.8 \pm 10.0$ & 0.87 & \\
\hline Diameter of lymph node. radiologically (mm) & $18.6 \pm 7.7$ & $20.7 \pm 10.2$ & $21.0 \pm 7.3$ & 0.41 \\
\hline Suv max of lymph node (Pet-CT) & $9.8 \pm 5.2$ & $7.8 \pm 4.5$ & $8.7 \pm 5.7$ & 0.28 \\
\hline Diameter of lymph node. EBUS (mm) & $20.4 \pm 8.4$ & $20.9 \pm 9.6$ & $21.6 \pm 8.3$ & 0.92 \\
\hline Number of Sampling & $3.6 \pm 1.0$ & $3.6 \pm 0.9$ & $3.5 \pm 1$ & 0.85 \\
\hline
\end{tabular}

endobronchial lesion was detected in 40 (14.8\%) patients. The most common indication for undergoing the EBUS-TBNA procedure was tumor staging $(44.1 \%)$.

\section{Characteristics of Patient Groups}

No statistically significant differences were found among the three patient groups in terms of age ( $\mathrm{p}=$ 0.87 ), radiologically measured lymph node diameter $(\mathrm{p}=0.41)$, PET-CT lymph node SUVmax value $(\mathrm{p}=0.28)$, lymph node diameter measured during EBUS-TBNA ( $p=0.92)$, or the number of lymph node samples taken $(\mathrm{p}=0.85)$ (Table 2$)$. Groups were also found to be similar in terms of age, radiological lymph node diameter, lymph node diameter measured during EBUS-TBNA, PET-CT lymph node SUVmax value, and the number of lymph node samples taken ( $p>0.05$ for all parameters). No differences among case groups were found by gender $(\mathrm{p}=0.38)$, presence of endobronchial lesions $(p=0.31)$, presence of a mass lesion in the lung $(\mathrm{p}=0.17)$, or indication for the EBUS-TBNA $(\mathrm{p}=0.47)$ (Table 3). Patients were also similar in terms of gender, endobronchial lesion status, presence of a mass lesion in the lung, and the indication for the procedure $(\mathrm{p}=0.05)$.

\begin{tabular}{|c|c|c|c|c|c|c|c|c|}
\hline \multirow[t]{3}{*}{ Measurement } & & \multicolumn{4}{|c|}{ Case Groups } & & & \multirow[t]{3}{*}{ p } \\
\hline & & \multicolumn{2}{|c|}{$\begin{array}{l}\text { Cases 1-90 } \\
(n=90)\end{array}$} & \multicolumn{2}{|c|}{$\begin{array}{l}\text { Cases } 91-180 \\
(n=90)\end{array}$} & \multicolumn{2}{|c|}{$\begin{array}{l}\text { Cases 181-270 } \\
(n=90)\end{array}$} & \\
\hline & & $\mathbf{n}$ & $\%$ & $\mathbf{n}$ & $\%$ & $\mathbf{n}$ & $\%$ & \\
\hline \multirow[t]{2}{*}{ Gender } & Female & 24 & $26.67 \%$ & 25 & $27.78 \%$ & 30 & $33.33 \%$ & 0.38 \\
\hline & Male & 66 & $73.33 \%$ & 65 & $72.22 \%$ & 60 & $66.67 \%$ & \\
\hline \multirow[t]{2}{*}{ Endobronchial } & No & 74 & $82.22 \%$ & 78 & $86.67 \%$ & 75 & $83.34 \%$ & 0.31 \\
\hline & Yes & 16 & $17.78 \%$ & 12 & $13.33 \%$ & 15 & $16.66 \%$ & \\
\hline \multirow[t]{2}{*}{ Mass lesion of lung in CT } & No & 35 & $38.89 \%$ & 41 & $45.56 \%$ & 39 & $43.33 \%$ & 0.17 \\
\hline & Yes & 55 & $61.11 \%$ & 49 & $54.44 \%$ & 51 & $56.67 \%$ & \\
\hline \multicolumn{9}{|l|}{ Indication for procedure } \\
\hline \multicolumn{2}{|l|}{ Diagnosis } & 20 & $22.22 \%$ & 30 & $33.33 \%$ & 30 & $33.33 \%$ & 0.47 \\
\hline \multicolumn{2}{|l|}{ Staging } & 38 & $42.22 \%$ & 41 & $45.56 \%$ & 40 & $44.44 \%$ & \\
\hline \multicolumn{2}{|c|}{ Diagnosis and Staging } & 5 & $5.56 \%$ & 0 & $0.00 \%$ & 3 & $3.33 \%$ & \\
\hline \multicolumn{2}{|c|}{ Studying Molecular Test } & 7 & $7.78 \%$ & 2 & $2.22 \%$ & 2 & $2.22 \%$ & \\
\hline \multicolumn{2}{|c|}{$\begin{array}{l}\text { Extra-thoracic Metastasis } \\
\text { of malignancy }\end{array}$} & 17 & $18.89 \%$ & 15 & $16.67 \%$ & 14 & $15.56 \%$ & \\
\hline \multicolumn{2}{|c|}{ Recurrence/Re-staging } & 3 & $3.33 \%$ & 2 & $2.22 \%$ & 1 & $1.11 \%$ & \\
\hline
\end{tabular}


International Journal of Hematology and Oncology

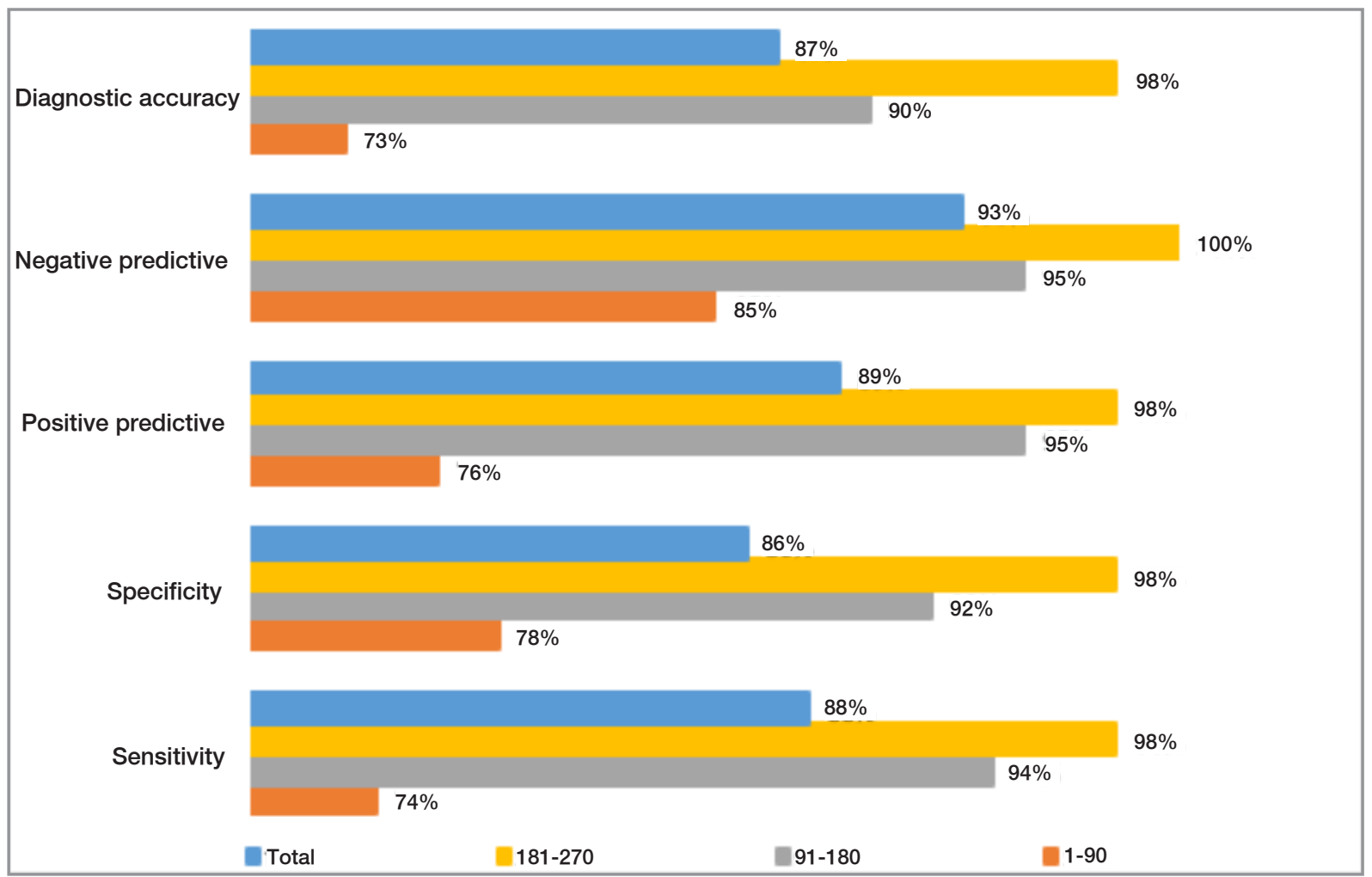

Figure 1. Comparison of all cases and groups

\section{Analysis of Diagnostic Accuracy by Patient Groups}

The diagnostic accuracy of EBUS-TBNA was compared among three patient groups (Table 4, Figure 1). ROC analysis revealed that the study bronchoscopist'sdiagnosis of malignancy based on EBUS-TBNA images were consistent with the final pathologist's diagnosis in $73 \%$ of the first 90 cases, $90 \%$ of the second 90 cases, and $98 \%$ of the third 90 cases. In the first 90 cases, the sensitivity of the bronchoscopist's diagnosis was $74 \%$, the specificity was $78 \%$, the positive predictive value was $76 \%$, and the negative predictive value was $85 \%$. In the second 90 cases, the sensitivity was
$94 \%$, the specificity was $92 \%$, the positive predictive value was $95 \%$, and the negative predictive value was $95 \%$. In the third 90 cases, the sensitivity was $98 \%$, the specificity was $98 \%$, the positive predictive value was $98 \%$, and the negative predictive value was $100 \%$. Thus, as the chronological order of patients increased, the accuracy of the bronchoscopist's diagnosis increased significantly.

\section{Complications}

Aside from minor bleeding that did not require intervention, no complications related to the procedure were reported.

\begin{tabular}{|llllll|}
\hline \multicolumn{7}{|l|}{ Table 4. Evaluation of diagnostic accuracy according to groups } \\
\hline Groups & Sensitivity & Specificity & Positive Predictive & Negative Predictive & Diagnostic Accuracy \\
\hline 1.-90. $(n=90)$ & $74 \%$ & $78 \%$ & $76 \%$ & $85 \%$ & $73 \%\left(p=0.02^{\star}\right)$ \\
$91-180(n=90)$ & $94 \%$ & $92 \%$ & $95 \%$ & $95 \%$ & $90 \%\left(p=0.01^{\star}\right)$ \\
$181-270(n=90)$ & $98 \%$ & $98 \%$ & $98 \%$ & $100 \%$ & $98 \%\left(p=0.01^{\star}\right)$ \\
Total $(n=270)$ & $88 \%$ & $86 \%$ & $89 \%$ & $93 \%$ & $87 \%\left(p=0.01^{\star}\right)$ \\
\hline PPV: positive predictive value; NPV: negative predictive value &
\end{tabular}




\section{DISCUSSION}

In this study, we found that as the number of EBUS-TBNA procedures performed by a single operator increased, so did the diagnostic accuracy of the procedure. While diagnostic accuracy was only moderate in the first 90 cases (73\%), it approached $100 \%$ in the last 90 patients. We showed that a physician, who is an expert in his field, needed to conduct at least 180 EBUS-TBNA procedures to acquire a high level of accuracy in diagnosis of lung malignancies. Our study showed that between the cases 91 and 180, the acceptable diagnostic accuracy rates were reached and these rates can increase with further operator experience.

Studies report diverse findings regarding the number of prior procedures that an operator should perform to reach an acceptable rate of diagnostic accuracy. Some studies report that sufficient diagnostic accuracy may be obtained after conducting ten procedures, however, most studies report that many more procedures, between 60 to 200 , must be conducted by an operator to acquire sufficient diagnostic accuracy. ${ }^{3,9-12}$ Similar to our findings, other studies report increased sensitivity in detection of malignancy as operator experience increases. In another study, EBUS-TBNA had a sensitivity of $64 \%$ in the first 37 patients assessed by the operator with EBUS-TBNA, compared to a sensitivity of $88.5 \%$ after the operator had assessed 62 patients. ${ }^{13}$ One study estimated that after conducting 212 procedures an operator may reach an expert level.

In present study, we were able to identify the impact of operator experience on diagnostic accuracy because EBUS-TBNA was performed by a single bronchoscopist and compared to a final diagnosis determined by a single pathologist. However, our findings regarding operator experience are intended to be generalized to EBUS-TBNA in highly trained specialist physicians. The study bronchoscopist had over ten years of conventional bronchoscopy experience as well as two months of training on EBUS-TBNA, during which he observed EBUSTBNA in 40 patients and performed the procedure himself in 40 patients, with an accompanying expert. Other studies also report that mastery of EBUS-TBNA is enhanced by specialized training and supervision. In a study conducted by Fernan-
dez-Villar et al. ${ }^{9}$, two operators, each with more than ten years of conventional bronchoscopy experience, underwent extensive, expert-led training on EBUS-TBNA; the operators'rates of diagnostic accuracy rate increased from $70 \%$ to $89.9 \%$ after the first 80 procedures. This study concluded that operators should perform at least 60 procedures to acquire adequate mastery of lymph node sampling, and perform at least 100 procedures to obtain a high level of mastery. Another study, in which operators did not receive specialized EBUS-TBNA training or have expert supervision, concluded that an operator should perform at least 60 procedures to achieve competency. ${ }^{14}$ These studies suggest that diagnostic accuracy rate increases both with the number of procedures performed under specialist supervision, as well as with the total number of procedure performed.

Lymph node size is one of the most important factors influencing diagnostic accuracy in conventional bronchoscopy. ${ }^{15}$ For example, in a multicenter study evaluating lung malignancy diagnosis by EBUS, the rates of diagnostic accuracy in the first 100 cases were found to be low (ranging from $59.7 \%$ to $80.3 \%$ ), which the authors attribute to initially sampling subcentimetric lymph nodes. ${ }^{3}$ In our study, the mean size of the lymph nodes was slightly higher than two centimeters, which may have contributed to our finding of high rates of diagnostic accuracy. In the study of Lin et al. in which all mediastinal and hilar nodes which are suspected to be pathologic were sampled, they reported that the learning curve drew an " $\mathrm{S}$ " pattern (which is described as "The initial curve rose slowly or even dropped, followed by a steeper ascending phase") and the learning curve improved after 120 cases. $^{16}$ This study included unselected patients, in which both benign and malign patients were taken, as our study.

The cytopathologist accompanied during the procedure for all patients and ROSE was done during the procedure. While the sampling process was ongoing, the cytopathologist gave instant feedback to the operator whether the sample was sufficient and whether it was taken from the correct place. We thinks that our diagnotic accuracy rates are high due to the factors that lymph nodes with a diameter of $10 \mathrm{~mm}$ and higher were sampled, cytopathologist waited for sufficient samples and made ROSE. 
Also, our sample numbers were high to enable the cytopathologist to have the most possible number of samples for molecular tests.

In result, in our study, an acceptable diagnostic accuracy rate was achieved with EBUS-TBNA after fewer procedures than reported in the literature. This result can be attributed to the facts that a single operator performed all EBUS-TBNA procedures, the operator received two months of EBUS-TBNA training before performing the procedures on his own, and all samples were evaluated by the same cytopathologist. We observed that as the number of EBUS-TBNA procedures performed by the operator increased, the diagnostic accuracy rates approached to perfection.

\section{REFERENCES}

1. Bray F, Ferlay J, Soerjomataram I, et al. Global cancer statistics 2018: GLOBOCAN estimates of incidence and mortality worldwide for 36 cancers in 185 countries. CA Cancer J Clin 68: 394-424, 2018.

2. Fuso L, Varone F, Magnini D, et al. Influence of the Learning Effect on the Diagnostic Yield of Endobronchial Ultrasoundguided Transbronchial Needle Aspiration of Mediastinal and Hilar Lymph Nodes. J Bronchology Interv Pulmonol 24: 193199, 2017.

3. Kemp SV, El Batrawy SH, Harrison RN, et al. Learning curves for endobronchial ultrasound using cusum analysis. Thorax 65: 534-538, 2010.

4. Zhu T, Zhang X, Xu J, et al. Endobronchial ultrasound guidedtransbronchial needle aspiration vs. conventional transbronchial needle aspiration in the diagnosis of mediastinal masses: A meta-analysis. Mol Clin Oncol 2: 151-155, 2014.

5. Herth FJF, Rabe KF, Gasparini S, Annema JT. Transbronchial and transoesophageal (ultrasound-guided) needle aspirations for the analysis of mediastinal lesions. Eur Respir J 28: 12641275, 2006.

6. Ernst A, Silvestri GA, Johnstone D, American College of Chest Physicians. Interventional pulmonary procedures: Guidelines from the American College of Chest Physicians. Chest 123: 1693-1717, 2003.

7. Steinfort DP, Hew MJ, Irving LB. Bronchoscopic evaluation of the mediastinum using endobronchial ultrasound: a description of the first 216 cases carried out at an Australian tertiary hospital. Intern Med J 41: 815-824, 2011.

8. Goldstraw P, Chansky K, Crowley J, et al. The IASLC Lung Cancer Staging Project: Proposals for Revision of the TNM Stage Groupings in the Forthcoming (Eighth) Edition of the TNM Classification for Lung Cancer. J Thorac Oncol 11: 3951,2016
9. Fernández-Villar $\mathrm{A}$, Leiro-Fernández $\mathrm{V}$, Botana-Rial $\mathrm{M}$, et al. The endobronchial ultrasound-guided transbronchial needle biopsy learning curve for mediastinal and hilar lymph node diagnosis. Chest 141: 278-279, 2012.

10. Davoudi M, Colt HG, Osann KE, et al.Endobronchial ultrasound skills and tasks assessment tool: assessing the validity evidence for a test of endobronchial ultrasound-guided transbronchial needle aspiration operator skill. Am J Respir Crit Care Med 186: 773-779, 2012.

11. Medford ARL. Learning curve for endobronchial ultrasoundguided transbronchial needle aspiration. Chest 141: 1643, 2012.

12. Stather DR, Chee A, MacEachern P, et al. Endobronchial ultrasound learning curve in interventional pulmonary fellows: Endobronchial ultrasound learning curve. Respirology 20: 333-449, 2015.

13. Usluer O, Kaya SO. Endobronchial ultrasound-guided transbronchial needle aspiration of mediastinal lymphadenopathy: effect of the learning curve. Interact Cardiovasc Thorac Surg 19: 693-695, 2014.

14. Kheir F, Alokla K, Myers L, Palomino J. Endobronchial ultrasound-transbronchial needle aspiration of mediastinal and hilar lymphadenopathy learning curve. Am J Ther 23: e10161019, 2016.

15. Bonifazi M, Zuccatosta L, Trisolini R, et al. Transbronchial needle aspiration: A systematic review on predictors of a successful aspirate. Respiration 86: 123-134, 2013.

16. Lin CK, Lai CL, Chang LY, et al. Learning curve and advantages of endobronchial ultrasound-guided transbronchial needle aspiration as a first-line diagnostic and staging procedure. Thorac Cancer 9: 75-82, 2018.

\section{Corresondence: \\ Dr. Fatih UZER \\ Kastamonu Devlet Hastanesi \\ Gögus Hastaliklari Bolumu \\ Merkez \\ KASTAMONU / TURKEY \\ Tel: (+090-554) 4811422 \\ e-mail: uzerfatih@gmail.com}

\section{ORCIDs:}

Rusen Uzun

0000-0001-6575-5066

Fatih Uzer

0000-0001-9318-0458 